\title{
Dark and Light Neurons in the Human Brain, with Special Reference to Their Reactions to Golgi's Silver Nitrate, Luxol Fast Blue MBS and Azocarmine G
}

\author{
Takuro Murakami ${ }^{1}$, Tetsuro Murakami ${ }^{2}$, Nazira Mahmut ${ }^{1,3}$, Sayoko Hitomi ${ }^{1}$ and Aiji Ohtsuka ${ }^{1}$ \\ Departments of Anatomy ${ }^{1}$, Neurology ${ }^{2}$ and Bacteriology ${ }^{3}$, Okayama University School of Medicine, Okayama, Japan
}

Received May 19, 1997

Summary. Sections from the human somatosensory cortex were observed with a light microscope. The neurons were classified into light and dark ones. The light neurons were slightly stained with thionin, luxol fast blue MBS and azocarmine $\mathrm{G}$ ( $80 \%$ of all neurons). The dark neurons were more or less shrunken, and stained intensely with these dyes (20\% of all neurons). Double staining with luxol fast blue MBS and azocarmine $G$ was especially useful to demonstrate the dark neurons, since it clearly stained even their fine processes. Neither light nor dark neurons were reactive to nick end labeling for detection of DNA fragmentation. Triple staining with Golgi's silver nitrate (or Gallyas's ammoniacal silver carbonate), luxol fast blue MBS and azocarmine $G$ showed that the majority of the dark neurons were argyrophilic (argyrophilic dark neurons, $15 \%$ of all neurons), while some of them were not argyrophilic (non-argyrophilic dark neurons, $5 \%$ of all neurons). Triple staining also showed that the light neurons were only occasionally argyrophilic (argyrophilic light neurons, $5 \%$ of all neurons); usually, the light neurons were not argyrophilic (non-argyrophilic light neurons, $75 \%$ of all neurons). The results confirm that dark neurons usually represent certain populations of neurons in the human brain, and that they are basically identical to the argyrophilic neurons. The discussion suggests that the argyrophilic light and dark neurons are excited cells, the non-argyrophilic dark neurons are exhausted cells, and the non-argyrophilic light neurons are resting cells. Triple staining further demonstrated that some glial cells were darkened and stained with Golgi's silver nitrate or Gallyas's silver carbonate. Additional Golgi's silver block staining showed that the argyrophilic neurons stained by the conventional block staining method usually possessed a shrunken cell body, which was characteristic of the dark neurons.
The occurrence in the brain and spinal cord of dark neurons characterized by their shrunken cell body and also by their unusually high affinity for histological stains has long been noted (FLESCH, 1887; RAMÓN Y CAJAL, 1909). The dark neurons are intensely stained with thionin, hematoxylin, pyronin, nuclear fast red, nile blue, gallocianin and certain other dyes such as carmine and erythrosine (Miller, 1949; TEWARI and BOURne, 1963; MAEDA, 1966).

Earlier researchers regarded the dark neurons as active cells (HODGE, 1892 cited by RAMÓN Y CAJAL, 1909) or described them as resting cells (RAMÓN Y CAJAL, 1909). However, modern authors have generally ascribed the occurrence of dark neurons to artifacts such as inadequate tissue fixation (FRIEDE, 1963; Ebels, 1975; AuER et al., 1985; GARMAN, 1990; LDBERG and TORVIK, 1993) or pathological changes after electric shock, ischemic attack, concussion, incision and other stimuli, including the administration of some toxins such as verotoxin (MAEDA, 1966; JOHNSON, 1975; QUEIROZ and FARIA, 1978; INGVAR et al., 1988; Dietrich et al., 1991; GALlyas et al., 1992a-c, 1993a, b; NACHEMSON and BENNETT, 1993; Islam et al., 1994: FeRČÁKOVÁ et al., 1955; MizuguCHI et al., 1996). In contrast, our recent studies have confirmed the usual appearance of dark neurons in the normal human, rat and mouse brains, and disclosed in the rat and mouse brains that the dark neurons usually observed in the normal brain and spinal cord are functionally induced by the circadian rhythm (MURAKAMI et al., 1993a, 1995b; MURAKAMI and OHTSUKa, 1995, 1996a; OHTSUKA and Murakami, 1996).

It has been recently reported that dark neurons experimentally or pathologically induced by brain concussion, arterial occlusion or cardiac arrest are 
preferentially stained with Gallyas's or Nauta's ammoniacal silver carbonate (GALLYAS et al., 1990, 1992a-c, 1993a, b; CzURKó and NisHINO, 1993; NISHINO et al., 1994; FERČÁKOVÁ et al., 1995). The present study reinvestigates human samples, to confirm the usual appearance of dark neurons in the brain and examine how these usually occurring or functionally induced dark neurons are reactive to silver stainings. The present study also discusses the usefulness of double staining with luxol fast blue MBS and azocarmine $G$ in staining the dark neurons.

\section{MATERIALS AND METHODS}

Some previously isolated human brain blocks $(3 \times 4 \times$ $4 \mathrm{~mm}$-blocks, somatosensory cortex-Area 7 of Brodmann, removed in surgery together with the meningioma from a 67-year-old Japanese man) (MURAKAMI et al., 1996c) were used in the present study. Briefly, the blocks had been fixed with $4.0 \%$ paraformaldehyde by puncture perfusion, and embedded in paraffin. Sections, $10-15 \mu \mathrm{m}$ in thickness, were prepared from these blocks, deparaffinized and stained as follows.

\section{Histological staining}

Some sections were stained with cationic iron colloid $(\mathrm{pH}$ 1.5) and carbol-thionin or with cationic iron colloid and nuclear fast red (MURAKAMI et al., 1986, 1993), and observed with a light microscope. After this, the sections were washed in distilled water, stained with azocarmine G (HEIDENHAIN, 1915) or luxol fast blue MBS (KLÜVER and BARRERA, 1953), and again observed with a light microscope.

Several sections were successively stained with luxol fast blue MBS and azocarmine G (double staining). In these cases, the cationic iron colloid staining was omitted.

\section{Silver staining}

Some sections were stained with silver nitrate by Golgi's original method (RomeIs, 1968). Briefly, the specimens were refixed with a mixture of $1.0 \%$ osmic acid and $2.5 \%$ potassium dichromate overnight, washed in distilled water for $6 \mathrm{~h}$, immersed in distilled water containing $0.75 \%$ silver nitrate overnight, and washed in distilled water for $1 \mathrm{~min}$. The sections were then immersed in a mixture of $0.3 \%$ hydroquinone and $20 \%$ formalin for $6 \mathrm{~h}$ in order to reduce impregnated silver nitrate.

Several sections were esterified, and treated with ammoniacal silver carbonate by the method by Gallyas (GALLYAS et al., 1990).
The silver-impregnated specimens, including those treated by the Golgi's method, were observed with a light microscope. After this observation, some sections were further stained with luxol fast blue MBS, azocarmine $\mathrm{G}$ or both of these dyes, and again observed with a light microscope.

\section{DNA nick end labeling}

Some sections were treated by the nick end labeling method for the detection of DNA fragmentation (GAVRIELI et al., 1992; NiTATORI et al., 1995), and observed with a light microscope (OHTSUKA and MURAKAMI, 1996).

\section{Golgi's silver block staining}

A deparaffinized block was block-stained by the original Golgi's silver impregnation method (ROMEIS, 1968). Briefly, the block was immersed in a mixture of $1.0 \%$ osmic acid and $2.5 \%$ potassium dichromate for 3 days, washed in distilled water for $2 \mathrm{~h}$, and immersed in a $0.75 \%$ silver nitrate aqueous solution for 4 days. The block was then cut into $10-15 \mu \mathrm{m}$ thick sections, and observed with a light microscope.

\section{RESULTS}

\section{Histological staining}

The specimens examined contained the III-VI layers of the somatosensory cortex. They showed neither pathological changes in structure nor invasions of tumor cells.

Many neurons in the III-VI layers possessed intensely negatively charged surface coats (perineuronal sulfated proteoglycans) which were reactive to cationic iron colloid and recognized as fine meshworks (Figs. 1A, 2). These surface coats surrounded the roots of cell processes as well as the cell bodies (Figs. 1A, 2). Glial cells never possessed such surface coats.

The majority of neurons were stained slightly with thionin, nuclear fast red, azocarmine $G$ and luxol fast blue MBS (about $80 \%$ of all neurons) (Figs. 1A, 2, 2 upper Inset). The nucleolus and nucleolus-associated chromatin of these light neurons were well stained with these dyes, especially with nuclear fast red and azocarmine $G$ (Fig. 2, upper Inset).

Many neurons, including some neurons with perineuronal sulfated proteoglycans, were chromophilic. These chromophilic or dark neurons were encountered in about $20 \%$ of all neurons in each layer, and randomly distributed among the light neurons (Fig. 1A, B, Insets). The cytoplasm of the dark neurons 


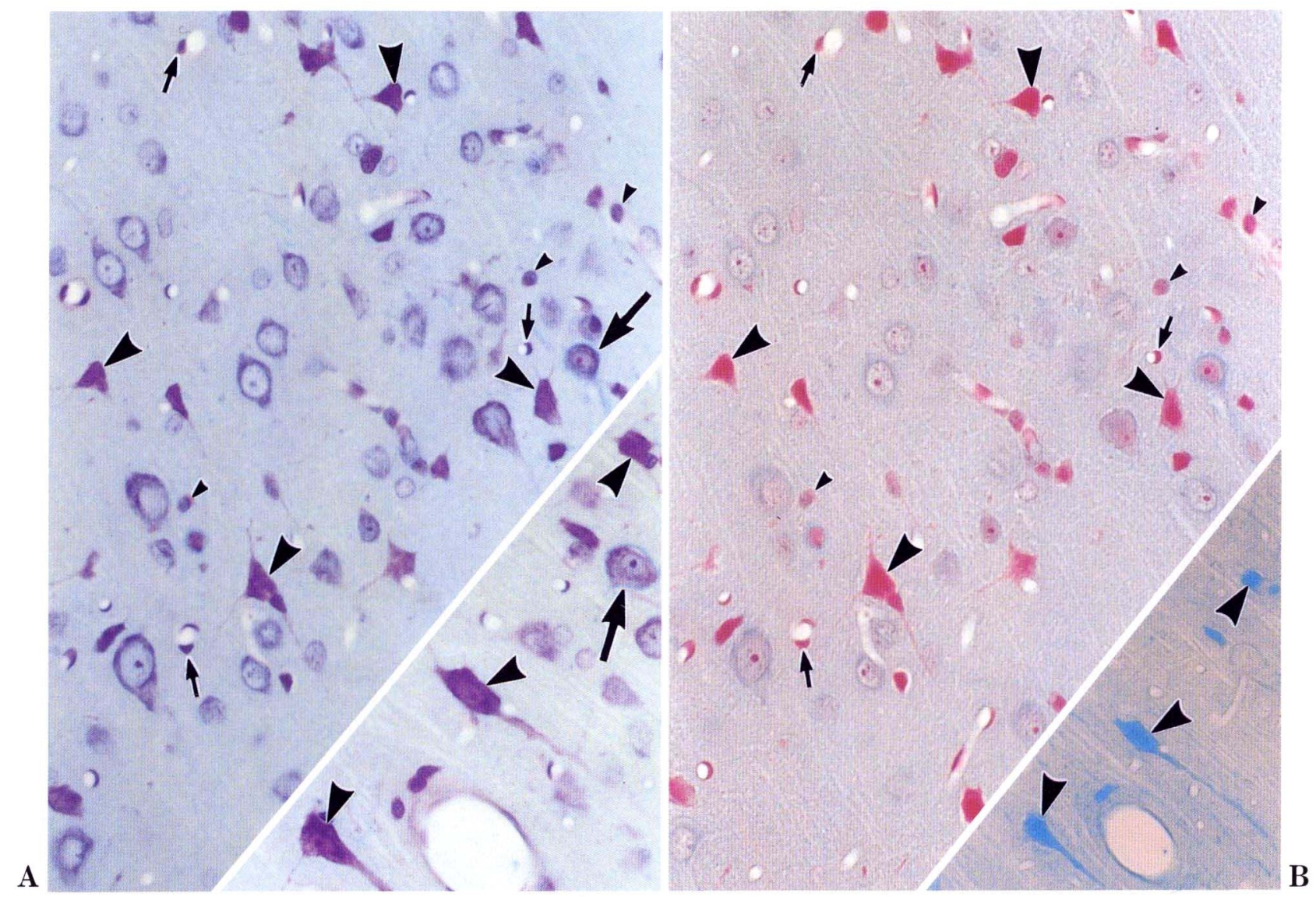

Fig. 1 A and Inset in A. Sections from the human somatosensory cortex, treated with cationic iron colloid ( $\mathrm{pH}$ 1.5) and thionin. Dark neurons are stained intensely with thionin (large arrowheads). Some neurons are coated with perineuronal sulated proteoglycans reactive to cationic iron colloid (large arrow). Some glial (small arrowheads) and capillary endothelial (small arrows) cells are also intensely stained with thionin. B shows the same section in $\mathbf{A}$, which was additionally stained with azocarmine G. Inset in $\mathbf{B}$ shows the same section in the Inset in $\mathbf{A}$, additionally stained with luxol fast blue MBS. Thionin was replaced with azocarmine G or luxol fast blue MBS, so that the dark neurons (large arrowheads) are stained solely with azocarmine G in $\mathbf{B}$ or with luxol fast blue MBS in Inset in B. Thionin-staining of the darkened glial (small arrowheads) and capillary endothelial (small arrows) cells was also replaced (A and B). A, Inset in A, B: $\times 260$, Inset in B: $\times 200$

was well reactive to thionin, nuclear fast red, luxol fast blue MBS and azocarmine G (Figs. 1A, B, 2). The nucleus, nucleolus and also nucleolus-associated chromatin of the dark neurons were also well reactive to these dyes (Fig. 2 lower Inset).

The dark neurons appeared more or less shrunken in their cell body. The perineuronal sulfated proteoglycans of such shrunken dark neurons were thickened, representing hairy meshworks (Fig. 2, lower Inset).

The cytoplasm and nucleus of the dark neurons were doubly stained with luxol fast blue MBS and azocarmine G (Fig. 3). This double staining was so intense that it visualized even their darkened fine processes (Fig. 3). In the combined stainings with thionin and azocarmine $\mathrm{G}$ or with thionin and luxol fast blue MBS, the dark staining with thionin was erased by washing the sections with water so that the thionin was thoroughly replaced with azocarmine $G$ (Fig. 1A, B) or luxol fast blue MBS (Fig. 1A Inset, B Inset).

In some dark neurons, their perinuclear zone or Golgi's area was scanty (vacant) and remained unstained (Figs. 2, 3 Inset).

The glial and capillary endothelial cells, or their cytoplasm and nucleus, were occasionally darkened and intensely stained with thionin, luxol fast blue MBS, and other dyes (Fig. 1A, B). 

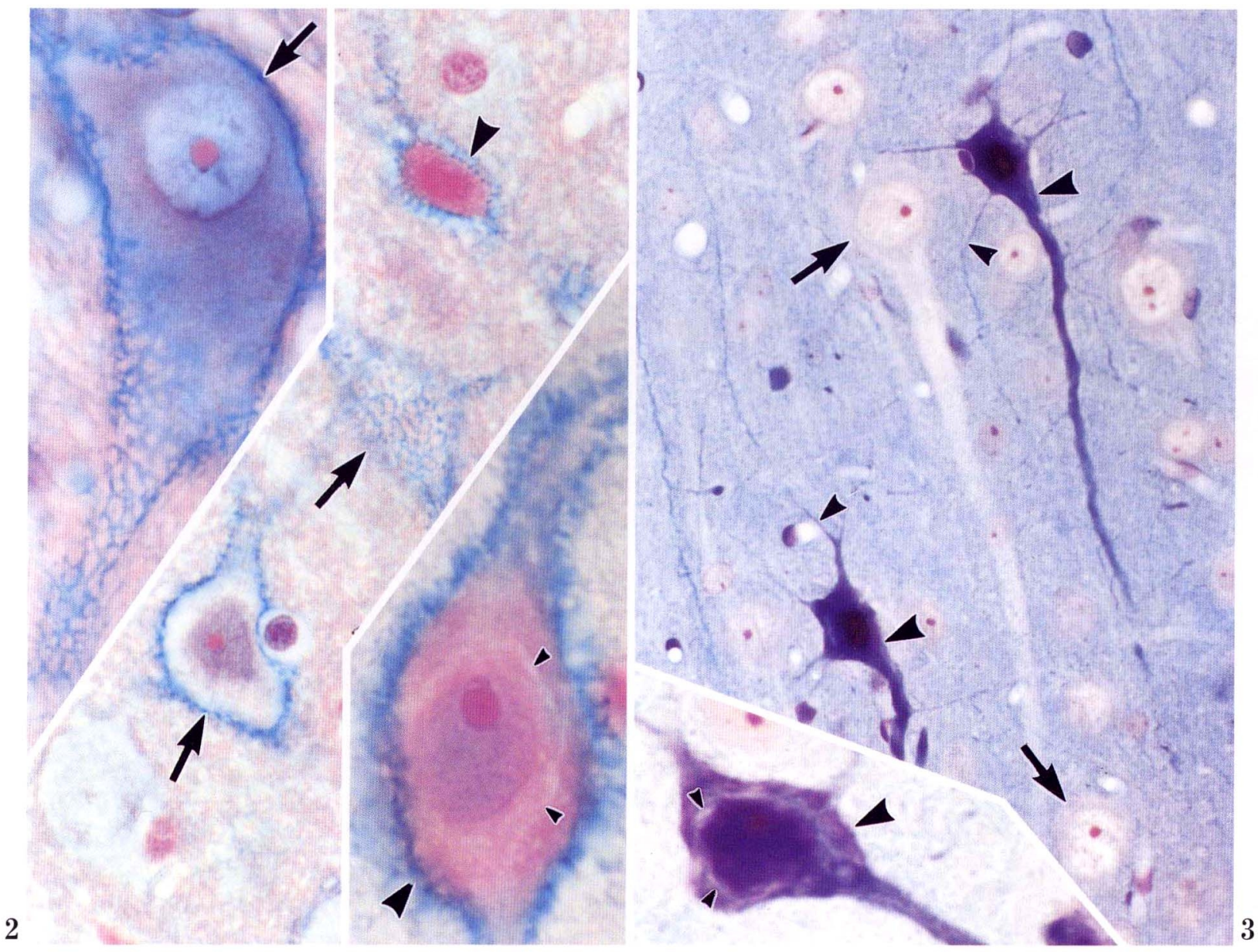

Fig. 2. Closer view of a section from the human somatosensory cortex, treated with cationic iron colloid (pH 1.5) and nuciear fast red. A neuron with perineuronal sulfated proteoglycans is darkened and intensely stained with nuclear fast red (large arrowhead). Large arrows indicate normal or light neurons with perineuronal sulfated proteoglycans. Upper inset shows a light neuron with well developed perineuronal proteoglycans (large arrow). Lower inset shows a dark neuron with perineuronal sulfated proteoglycans (large arrowhead). The perinuclear zone or Golgi's area of this neuron is scanty and remains unstained (small arrowheads). $\times 600$, Upper inset: $\times 1,300$, Lower inset: $\times 1,400$

Fig. 3. Section treated with luxol fast blue MBS and azocarmine G. Dark neurons (large arrowheads) and their processes, including fine ones (small arrowheads), are stained doubly with luxol fast blue MBS and azocarmine G. In contrast, the light neurons, except their nuclei, show no reaction to these dyes (large arrows). Inset shows a doubly stained dark neuron (large arrowhead) whose Golgi's area is scanty and remains unstained (small arrowheads). $\times 450$, Inset: $\times 1,000$

\section{Silver staining}

A considerable number of neurons in the III-VI layers of the human somatosensory cortex were stained with Golgi's silver nitrate (Fig. 4A) or Gallyas's ammoniacal silver carbonate (about $20 \%$ of all neurons) (Fig. 4A, A Inset). In the cell staining, no marked difference was noted between the Golgi's and Gallyas's methods. In both methods, therefore, the silver staining was recognized as fine granules diffusely deposited in the cytoplasm (Fig. 5, upper and lower Insets). Such granular silver deposits were also noted as dotted lines in the fine pocesses around or near the silver-impregnated cell bodies (Fig. 5).

The silver-treated sections were well reactive to luxol fast blue MBS and azocarmine G, when they were solely stained with either of these dyes (Fig. 5B Inset). When the silver-treated sections were stained doubly with luxol fast blue MBS and azocarmine G, azocarmine $\mathrm{G}$ staining was weakened or erased; in such cases, the cytoplasm and nucleus were mainly stained with luxol fast blue MBS, and the nucleolus 

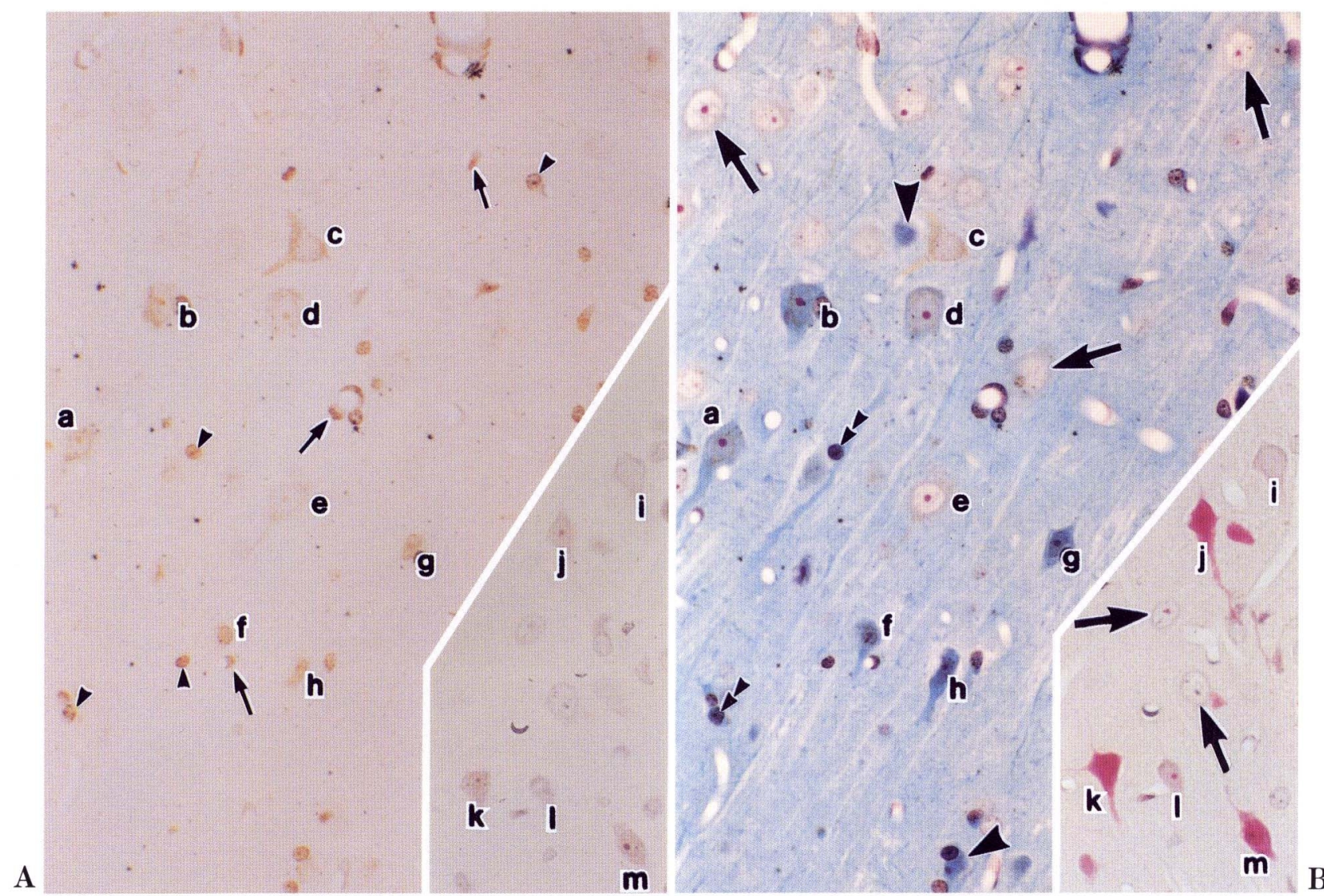

Fig. 4 A. Section treated with Golgi's silver nitrate. Many neurons are argyrophilic and reactive to silver nitrate $(a-h)$. Some glial (small arrowheads) and capillary endothelial (small arrows) cells are also reactive to silver nitrate. $\mathbf{B}$ shows the same section in A, additionally treated with luxol fast blue MBS and azocormine G. The $a, b, d, f-h$ neurons are doubly stained with silver nitrate and luxol fast blue MBS (argyrophilic dark neurons). The $c$ and $e$ neurons are solely reactive to silver nitrate (argyrophilic light neurons). The neurons indicated by large arrowheads are solely reactive to luxol fast blue MBS (non-argyrophilic dark neurons). Other neurons are stained neither with silver nitrate nor with luxol fast blue MBS (non-argyrophilic light neurons) (large arrows). The glial cells indicated by duplicated small arrowheads are doubly stained with luxol fast blue MBS and azocarmine G. Inset in A shows a section treated with Gallyas's ammoniacal silver carbonate. Some neurons are stained with this silver carbonate $(i-m)$. Inset in B shows the same section in Inset in A, additionally treated with azocarmine G. The $j, k$ and $m$ neurons are doubly stained with ammoniacal silver carbonate and azocarmine $\mathrm{G}$. The $i$ and $l$ argyrophilic neurons are not stained with azocarmine $\mathrm{G}$. A, B: $\times 200$, Insets: $\times 200$

and nucleolus-associated chromatin with azocarmine G (Figs. 5, 6).

According to their cytoplasmic reactions to silver (Golgi's silver nitrate and Gallyas's ammoniacal silver carbonate) and dyes (luxol fast blue MBS and azocarmine $\mathrm{G}$ ), the neurons could be classified into four types: 1) argyrophilic dark neurons doubly stained with silver and the dyes (about 15\% of all neurons) (Figs. 4B, B Inset, 6, upper Inset); 2) nonargyrophilic dark neurons solely stained with the dyes (about $5 \%$ of all neurons) (Figs. 4B, 6, upper and lower Insets); 3) argyrophilic light neurons solely stained with silver (about 5\% of all neurons) (Figs. 4B, B Inset, 5, upper and lower Insets, 6, upper Inset); and 4) non-argyrophilic light neurons stained neither with silver nor with the dyes (about $75 \%$ of all neurons) (Fig. 4B).

The glial and capillary endothelial cells, especially their nuclei, were frequently reactive to Golgi's silver nitrate or Gallyas's ammoniacal silver carbonate (Fig. 4A). These argyrophilic glial and endothelial cells were usually darkened and stained intensely with luxol fast blue MBS or azocarmine G (Figs. 5 left-lower Inset, 6). 

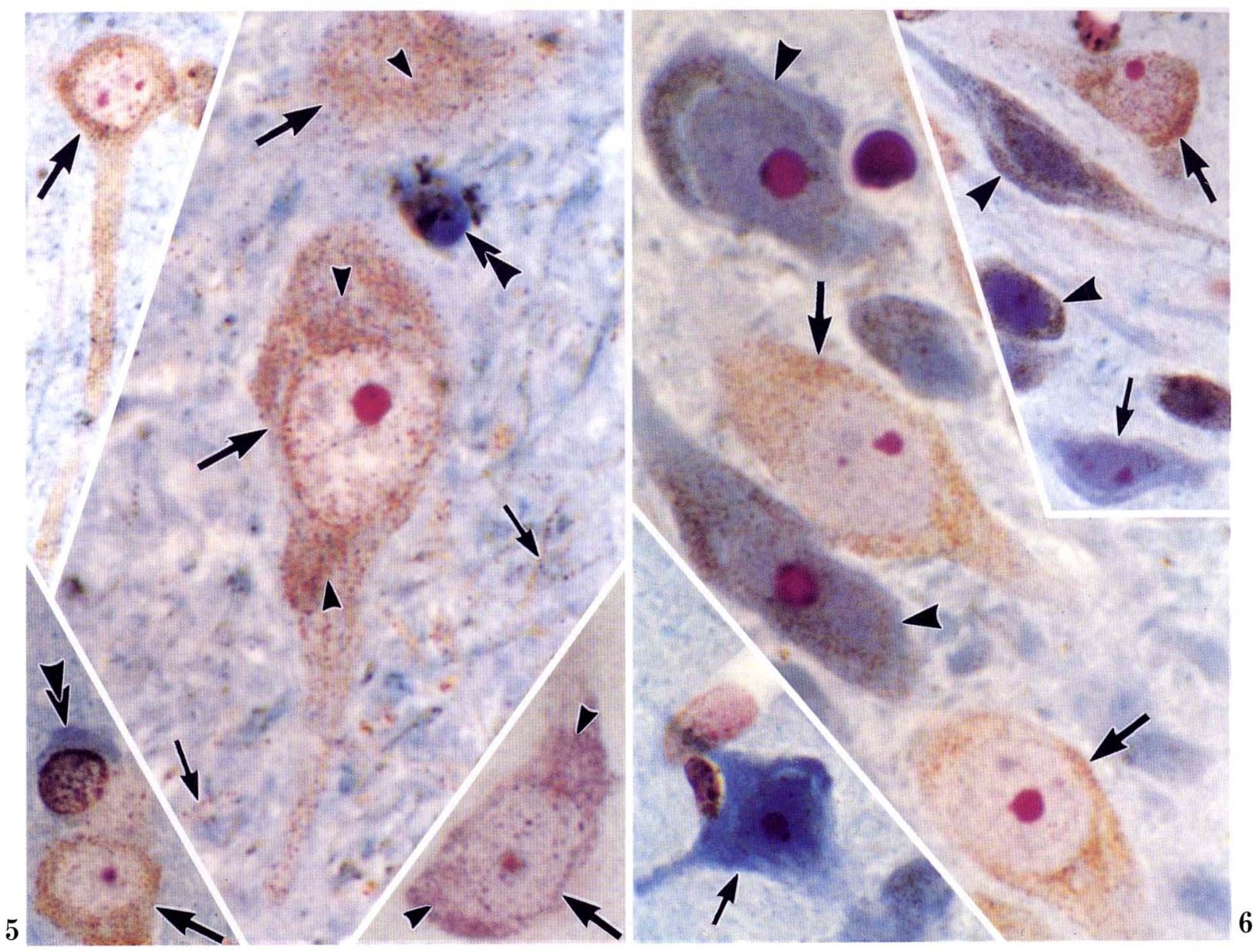

Fig. 5. Argyrophilic neurons as stained with Golgi's silver nitrate. This section was counter-stained with luxol fast blue MBS and azocarmine G. Golgi's silver staining is observed as granular deposits in the cytoplasm (small arrowheads). Such granular deposits in the fine processes are noted as dotted lines (small arrows). A glial cell both reactive to silver nitrate and luxol fast blue MBS is seen (duplicated large arrowheads). Upper inset shows another example of argyrophilic neuron stained with Golgi's silver nitrate (large arrow). Lower-left inset shows another example of glial cell both reactive to silver nitrate and luxol fast blue MBS (duplicated large arrowheads). In the glial cell, silver reaction mainly occurs in the nucleus. A neuron solely reactive to silver nitrate (large arrow) is also seen in this Inset. Lower-right inset shows an argyrophilic neuron stained with Gallyas's ammoniacal silver carbonate (large arrow)(counter-stained with azocarmine G). Even in the Gallyas's method, the silver staining was observed as the fine granular deposits in the cytoplasm (small arrowheads). $\times 1,200$, Upper inset: $\times 600$, Lower-left inset: $\times 650$, Lower-right inset: $\times 1,000$

Fig. 6, Upper and Lower Insets. Sections treated with Golgi's silver nitrate, luxol fast blue MBS and azocarmine G. Large arrowhead indicates the neuron doubly stained with silver nitrate and luxol fast blue MBS (argyrophilic dark neuron). Large arrow indicates the neuron stained solely with silver nitrate (argyrophilic light neuron). Small arrow indicates the neuron solely stained with luxol fast blue MBS (non-argyrophilic dark neuron). $\times 1,200$, Upper inset: $\times 750$, Lower inset: $\times 900$ 


\section{Nick end labeling}

No nick end labeling was noted in any neurons, including the dark ones (data, not shown). Neither was any nick end labeling noted in any glial or capillary endothelial cells.

\section{Golgi's silver block staining}

In the III-VI layers, some neurons and their processes were intensely impregnated with silver (Fig. 7, Inset). Many neurons were not stained with silver, and their cell bodies appeared as vacant structures with rather round forms (Fig. 7, Inset).

\section{DISCUSSION}

The present study, together with our previous studies of the human visual and somatosensory cortices (Murakami et al., 1993b, 1994b, 1995b, 1996c), confirms the occurrence of perineuronal sulfated proteoglycans in the human brain. These perineuronal proteoglycans are identical to Golgi's reticular coating (MURAKAMI et al., 1996c), and are widely recognized in the brain and spinal cord of the cow, dog, cat, rat, mouse and other animals (BRÜCKNER et al., 1993, 1996; MuraKami et al., 1993a, 1994a, 1996b; CELIO and BlÜMCKe, 1994; SEEgER et al., 1994). Their detailed distribution, development and morphological features have been dealt with elsewhere (MURAKAMI et al., 1995b, c, 1996b; Tsubouchi, Y. et al., 1996; Tsubouchi, M. et al., 1996).

The present study, together with our previous studies of the human visual cortex (MURAKAMI et al., 1993b, 1995b), confirms the usual appearance of dark neurons in the human brain. Negative labeling of DNA nick end in the dark neurons suggests that such dark profiles may not represent apoptosis. Our previous studies of the rat and mouse brains have also confirmed that the dark neurons are not reactive to DNA nick end labeling, and further disclosed that the dark neurons show a circadian rhythm in their occurrence. Neurons have been demonstrated to be darkened in the night when the animal is aroused, and restored to light cells during sleep or after resting of the cells in the day time (MURAKAMI and OHTSUKA, 1995, 1996a; Ohtsuka and Murakami, 1996).

The present study proves that luxol fast blue MBS and azocarmine $\mathrm{G}$ as well as thionin and nuclear fast red are useful dyes for staining the dark neurons. The double staining with luxol fast blue MBS and azocarmine $G$ is especially useful to demonstrate the

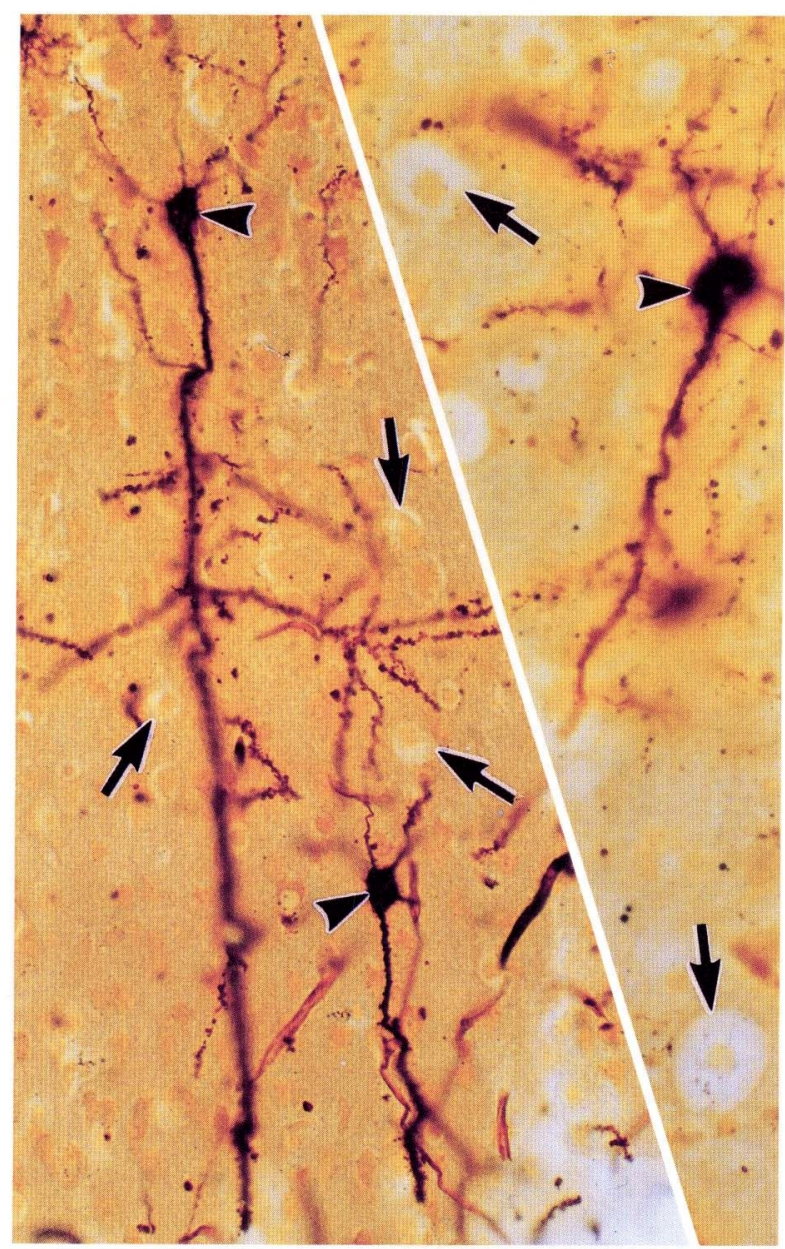

Fig. 7 and Inset. Sections from the specimen which was block-stained with Golgi's silver nitrate. Some neurons and their processes are intensely reactive to this silver nitrate (large arrowhead). Large arrows indicate the unstained neurons. $\times 260$, Inset: $\times 550$

dark neurons, since it clearly stains even their fine processes.

Noteworthy is that the nucleus and cytoplasm of the dark neurons are intensely reactive to nuclear fast red or azocarmine $\mathrm{G}$ with a specific affinity to the nucleolus. This may suggest an increased production of ribonucleic acids (mRNA) in the nucleus and also an increased supply of these nucleic acids to the cytoplasm. Similar findings were reported by TEWARI and BOURNE (1963), who stained the rabbit cerebellar Purkinje cells with methyl green and pyronin.

Also noteworthy is that the cytoplasm of the dark neurons is intensely reactive to luxol fast blue MBS with an affinity to phospholipids or glycerols. This may be closely associated with an increased accumulation of inositol phospholipids or diacylglycerols in 
the darkened cytoplasm, which are known to be produced richly in the excited nerve cells (ALBERTS et al., 1989, 1994).

The present study proves that the Golgi's original silver block impregnation method is useful to stain the sections from paraffin-embedded specimens, when an appropriate reduction procedure is inserted after the silver impregnation. It also proves that the Gallyas's ammoniacal silver carbonate method (GALLYAS et al., 1990) is compatible with the Golgi's silver nitrate method in staining the nerve cells. GoMORI (1932) reported that calcium which has reacted to phosphate groups could easily be replaced with Golgi's silver nitrate. This suggests an increased intracytoplasmic accumulation of calcium in the argyrophilic dark and light neurons. ISLAM et al. (1995) autoradiographically proved a rich calcium accumulation in the electrically stimulated or darkened rat brain neurons.

The present silver impregnation followed by luxol fast blue MBS or azocarmine G staining supplements the findings by GALLYAS et al. (1990, 1992a-c, 1993, 1994) and other authors (CZURKÓ and NISHINO, 1993; NiSHINO et al., 1994; FERČÁKOVÁ et al., 1995), and gives evidence that the dark neurons-due to the circadian rhythm - are not always argyrophilic, some of them being not reactive to silver-non-argyrophilic dark neurons. It also gives evidence that some of the light neurons are stained with silver (argyrophilic light neurons).

Not much data are available on the fine structures of the dark neurons (PETERS et al., 1991). Our recent electron microscope findings from the brain of aroused mice showed that the functionally induced dark neurons are characterized by marked features of dehydration (Murakami et al., 1996d). Dehydration may easily develop in the neurons since they mainly use glucose as energy source, which produces rather limited amounts of metabolic water (MAYES, 1993). Such cytoplasmic vacuolations, including the autophagic ones, as observed in the experimentally induced dark neurons (INGVAR et al., 1988; DIETRICH et al., 1991) or in the apoptotic neurons (IwANOWSKI, 1988), were never noted in the dark neurons due to the circadian rhythm (MURAKAMI et al., 1996d).

Our recent electron microscope observations of mouse brain sections showed that the dark neurons usually contained fully expanded Golgi's complexes with many vesicular elements (excited dark neurons), whereas some of them contained dilated Golgi's complexes and few vesicles (exhausted neurons) (MURAKAMI et al., 1996d). These dilated Golgi's complexes may be observed as scanty or unstained Golgi's areas (perinuclear zone) under a light microscope, as sometimes identified even in the human dark neurons studied here.

The present study additionally demonstrates that the neurons stained by the Golgi's block silver impregnation method usually possess a shrunken cell body, and that the argyrophilic dark neurons stained in the sections also frequently possess the same. This supports the view that the block-stained argyrophilic neurons are identical to those stained in the sections, and that majority of them are included in the dark neurons.

The present study further discloses that even the glial and capillary endothelial cells are darkened, and that they are usually argyrophilic. This indicates that the active and resting phases may also occur in these cells.

Acknowledgments. We are grateful to Assoc. Prof. Isao DATE for providing human tissues, and to Mr. Hiromichi Kusano, Mr. Masahiro NARASAKI, and Mr. Hisao Mizogu $\mathrm{CHI}$ for tissue preparation and staining. We are also grateful to Prof. Shigetoshi KURODA for introducing the Gallyas's silver impregnation method.

\section{REFERENCES}

Alberts, B., D. Bray, J. Lewis, M. RafF, K. Roberts and J. D. Whatson: The nervous system. In: (ed. by) B. AlBerts et al.: Molecular biology of the cell. 2nd ed. Garland Publishing, New York and London, 1989 (p. 1059-1136).

—: Molecular biology of the cell. 3rd ed. Garland Publishing, New York and London, 1994 (p. 721-785).

Auer, R. N., H. Kalimo, Y. Olsson and B. K. Siesjö: The temporal evolution of hypoglycemic brain damage: I. Light and electron microscopic findings in the rat cerebral cortex. Acta Neuropathol. 67: 13-24 (1985).

Brückner, G., K. Brauer, W. Härtig, J. R. WolfF, M. J. Rickmann, A. Derouiche, B. Delpech, N. Gigard, W. H. Oertel and A. Reichenbach : Perineuronal nets provide a polyanionic, glia-associated form of microenvironment around certain neurons in many parts of the rat brain. Glia 8: 183-200 (1993).

Brückner, G., W. Härtig, J. KaCZA, J. SeEger, K. WELT and K. BRAUER: Extracellular matrix organization in various regions of rat brain grey matter. J. Neurocytol. 25: 333-346 (1996).

Celio, M. R. and I. BLüMcke: Perineuronal nets. A specialized form of extracellular matrix in the adult nervous system. Brain Res. Rev. 19: 128-145 (1994).

Czurkô, A. and H. Nishino: 'Collapsed' (argyrophilic, dark) neurons in rat model of transient focal cerebral ischemia. Nerosci. Lett. 162: 71-74 (1993).

Dietrich, W. D., M. Halley, I. Valdes and R. Busto: Interrelationships between increased vascular perme- 
ability and acute neuronal damage following temperature-controlled brain ischemia in rats. Acta Neuropathol. 81: 615-625 (1991).

Ebels, E.: Dark neurons. A significant artifact: The influence of maturational stage of neurons on the occurrence of the phenomenon. Acta Neuropathol. 33: 271273 (1975).

Fer č ÁKovâ, A., I. VANickÝ, M. MAR Š ALA and J. MAR Š ALA: Effect of prolonged hyperventilation on ischemic injury of neurons after global brain ischemia in the dog. J. Brain Res. 36: 297-304 (1995).

Flesch, M.: Über die Verschiedenheiten im chemischen Verhalten der Nervenzellen. Mitt. Naturforsch. Ges. (Bern) 1073-1082: 192-199 (1887).

FRIEDE, R. L.: Interpretation of hyperchromic nerve cells. J. Comp. Neurol. 121: 137-149 (1963).

Gallyas, F., F. H. Güldner, G. Zoltay and J. R. WoLfF: Golgi-like demonstration of "dark" neurons with an argyrophil III method for experimental neuropathology. Acta Neuropathol. 79: 620-628 (1990).

Gallyas, F., G. Zoltay and I. Balas: An immediate light microscope response of neuronal somata, dendrites and axons to contusing concussive head injury in the rat. Acta Neuropathol. 83: 394-401 (1992a).

Gallyas, F., G. Zoltay and Z. HoRváth: Light microscopic response of neuronal somata, dendrites and axons to post-mortem concussive head injury. Acta Neuropathol. 83: 499-503 (1992b).

Gallyas, F., G. Zoltay and W. Dames: Formation of "dark" (argyrophilic) neurons of various origin proceeds with a common mechanism of biophysical nature (a novel hypothesis). Acta Neuropathol. 83: 504-509 (1992c)

Gallyas, F., M. Hsu and G. BuZsáki: Four modified methods for thick sections of formaldehyde-fixed mammalian central nervous tissue: 'dark' neurons, perikarya of all neurons, microglial cells and capillaries. J. Neurosci. Methods 50: 159-164 (1993a).

Gallyas, F., G. Zoltay, Z. HoRvâth, K. Dávid and L. KELÉNYI: An immediate morpholopathological response of neurons to electroshock: A reliable model for producing "dark" neurons in experimental neuropathology. Neurobiology 1: 133-146 (1993b).

GaRMan, R. H.: Artifacts in routinely immersion fixed nervous tissue. Toxicol. Pathol. 18: 149-153 (1990).

Gavrieli, Y., Y. Sherman and S. A. Ben-Sasson : Identification of programmed cell death in situ via specific labeling of nuclear DNA fragmentration. J. Cell Biol. 119: 493-501 (1992).

GOMoRI, G.: Der mikrotechnischen Nachweis unlöslich Kalzsalze in den Geweben. Virchows Arch. 286: 682-689 (1932).

Heidenhain, H.: Über die Mallorysche Bindegewebsfärbung mit Karmin and Azokarmine als Vorfarben. Z. Wissensch. Mikrosk. Technik 32: 361-372 (1915).

IngVar, M., P. F. Morgan and R. N. AuER: The nature and timing of excitotoxic neuronal necrosis in the cerebral cortex, hippocampus and thalamus due to flurothyl-induced status epilepticus. Acta Neuropathol.
75: 362-369 (1988).

Islam, N., A. Moriwaki, Y. Hattori and Y. HoRI: Appearance of dark neurons following anodal polarization in the rat brain. Acta Med. Okayama 48: 123-130 (1994).

Islam, N., M. Aftabuddin, A. Moriwaki, Y. Hattori and Y. HoRI: Increase in the calcium level following anodal polarization in the rat brain. Brain Res. 684: 206-208 (1995).

IwANowski, L.: Apoptosis and dark neurons. Neuropathol. (Pol.) 26: 573-579 (1988).

Johnston, J. E. JR.: The occurrence of dark neurons in the normal and deaffected lateral vestibular nucleus in the rat. Acta Neuropathol. 31: 117-127 (1975).

KLÜVER, H. and E. BARRERA: A method for the combined staining of cells and fibers in the nervous system. J. Neuropathol. Exp. Neurol. 12: 400-403 (1953).

LøberG, E. M. and A. Torvik: Distinction between artefactually shrunken and truly degenerated 'dark' neurons by in situ fixation with microwave irradiation. Neuropathol. Appl. Neurobiol. 19: 359-363 (1993).

MAEDA, T.: Experimental and histochemical studies on the hyperchromic neurons (Japanese text with English abstract). Arch. Histol. Jap. 27: 177-197 (1966).

Mayes, P. A.: Bioenergetics and the metabolism of carbohydrates and lipids. In: (ed. by) R. K. MurRAY et al.: Harper's Biochemistry. Prentice-Hall International, Conneticut, 1993 (p. 105-279).

Miller, R. A.: A morphological and experimental study of chromophilic neurons in the cerebral cortex. Amer. J. Anat. 84: 201-229 (1949).

Mizuguchi, M., S. Tanaka, I. FujiI, H. Tanizawa, Y. Suzuki, T. Igarashi, T. Yamanaka, T. Takeda and $M$. Miwa: Neuronal and vascular pathology produced by verocytotoxin 2 in the rabbit central nervous system. Acta Neuropathol. 91: 254-262 (1996).

Murakami, T., T. Taguchi, A. Ohtsuka, K. Sano, T. Kaneshige, R. L. Owen and A. L. Jones: A modified method of fine-granular cationic iron colloid preparation: Its use in the light and electron microscopic detection of anionic sites in the rat kidney glomerulus and certain other tissues. Arch. Hiostol. Jap. 49: 12-23 (1986).

Murakami, T., T. Taguchi, A. Ohtsuka and A. Kikuta: Neurons with strongly negative-charged surface-coats in adult rat brain as detected by staining with cationic iron colloid. Arch. Histol. Cytol. 56: 13-21 (1993a).

Murakami, T., T. Taguchi and A. Ohtsuka: The occurrence in the human brain of neurons with strongly negative-charged proteoglycans. Arch. Histol. Cytol. 56: 23-26 (1993b).

Murakami, T., Y. Tsubouchi, M. Tsubouchi, A. OhtsuKA, T. TAguchi and A. KikUTA: The occurrence of rat spinal cord neurons with strongly negative-charged surface coats. Arch. Histol. Cytol. 56: 501-504 (1993c).

Murakami, T., M. Tsubouchi, Y. Tsubouchi, T. TaguCHI and A. OHtsuka: The occurrence of neurons with strongly negatively charged surface coats in mammalian, avian, reptilian, amphibian and piscine brains. 
Acta Med. Okayama 48: 195-197 (1994a).

Murakami, T., A. Ohtsuka and T. Taguchi: Neurons with intensely negatively charged extracellular matrix in the human visual cortex. Arch. Histol. Cytol. 57: 509522 (1994b).

Murakami, T. and A. OHTSUKa: Sleep restores the ratio of dark neurons to light neurons in the central nervous system (Japanese text with English abstract). Okayama Igakkai Zasshi 107: 235-242 (1995a).

Murakami, T., S. Hitomi, A. Ohtsuka and T. Taguchi: Neurons with perineuronal sulfated proteoglycans in the human visual cortex, with special reference to their reactions to lectins. Arch. Histol. Cytol. 58: 357-364 (1995b).

Murakami, T., A. Ohtsuka, T. Taguchi and D. X. Pia0: Perineuronal sulfated proteoglycans and dark neurons in the brain and spinal cord: A histochemical and electron microscopic study of newborn and adult mice. Arch. Histol. Cytol. 58: 557-565 (1995c).

Murakami, T. and A. Ohtsuka: Sleep resusciates the central nervous system (In Japanese). Microscopia (Niigata) 13: 164-170 (1996a).

Murakami, T., A. Ohtsuka and K. Ono: Neurons with perineuronal sulfated proteoglycans in the mouse brain and spinal cord: Their distribution and reactions to lectin Vicia villosa agglutinin and Golgi's silver nitrate. Arch. Histol. Cytol. 59: 219-231 (1996b).

Murakami, T., A. Ohtsuka and D. X. Piao: Perineuronal sulfated proteoglycans in the human brain are identical to Golgi's reticular coating. Arch. Histol. Cytol. 59: 233-237 (1996c).

Murakami, T., A. Ohtsuka and S. Yamana: Dark and light neurons in the central nervous system: A light and electron microscopic study of the mouse brain (Japanese text with English abstract). Okayama Igakkai Zasshi 108: 303-310 (1996d).

NaChemson, A. K. and G. J. BennetT: Does pain damage spinal cord neurons? Transsynaptic degeneration in rat following a surgical incision. Neurosci. Lett. 162: 78-80 (1993).

Nitatori, T., N. Sato, S. Waguri, Y. Karasawa, H. Araki, K. Shibanai, E. Kominami and Y. UchiYama: Delayed neuronal death in the CA1 pyramidal cell layer of the gerbil hippocampus following transient ischemia is apoptosis. J. Neurosci. 15: 1001-1011 (1995).

Nishino, H., A. Czurkó, A. Fukuda, T. Hashitani, H. Hida, Z. KARAdi and L. LÊnÁRd: Pathophysiological process after transient ischemia of the middle cerebral artery in the rat. Brain Res. Bull. 35: 51-56 (1994).

Ohtsuka, A. and T. Murakami: Dark neurons in the mouse brain: An investigation into the possible significance of their variable appearance within a day and their relation to negatively charged cell coats. Arch. Histol. Cytol. 59: 79-85 (1996).
Peters, A., S. L. Palay and H. De F. Webster: The neuronal cell body. In: (ed. by) A. PETERS, L. PALAY and H. DE F. WEBSTER: The fine structure of the nervous system. Oxford University Press, New York-Oxford, 1991 (p. 14-69).

Queiroz, L. DE S. and J. Lopes DE FARIA: Evaluation of dark neurons in experimental brain stab wounds. Virchows Arch. B Cell Pathol. 28: 361-370 (1978).

RAMón Y CAJAL, S. (1909): Variations in the structure and shape of neurons under normal and pathological conditions, and the functional implications of such variations. In: (ed. by) N. Swanson and L. Swanson: Histology of the nervous system of man and vertebrates by $\mathrm{S}$. Ramón y Cajal. Vol. I. Oxford University Press, New York and Oxford, 1995 (p. 174-190).

Romeis, B.: Die Ausführung der Golgi-Original-Methoden. In: (ed. by) B. RomeIs: Mikroskopische Technik. 16. ed., R. Oldenbourg, München-Wien, 1968 (p. 428-430).

Seeger, G., K. Brauer, W. Härtig and G. BrüCKNer: Mapping of perineuronal nets in the rat brain stained by colloidal iron hydroxide histochemistry and lectin cytochemistry. Neuroscience 58: 371-388 (1994).

TeWARI, H. B. and G. H. Bourne: Histochemical studies on the "dark" and "light" cells of the cerebellum of rat. Acta Neuropathol. 3: 1-15 (1963).

Tsubouchi, M., Y. Tsubouchi, S. Hitomi, A. Ohtsuka and T. MUrakami: Perineuronal sulfated proteoglycans, cell surface glycoproteins and dark neurons in the cingulate cortex of the newborn and adult rats. Acta Med. Okayama 50: 313-317 (1996).

Tsubouchi, Y., M. Tsubouchi, S. Hitomi, A. Ohtsuka and T. Murakami: Perineuronal sulfated proteoglycans in the adult rat brain: Histochemical and electron microscopic studies. Acta Med. Okayama 50: 237241 (1996).

\author{
Prof. Takuro MuRAKAMI \\ Department of Anatomy \\ Okayama University School of Medicine \\ 2-5-1 Shikata-cho, Okayama \\ 700 Japan \\ 村上 宅 郎 \\ 700 岡山市鹿田町 2-5-1 \\ 岡山大学医学部 \\ 解剖学第二講座
}

